Pathologe 2021 - 42 (Suppl 2):S199-S202 https://doi.org/10.1007/s00292-021-00991-0 Angenommen: 3. September 2021 Online publiziert: 5 . Oktober 2021 (c) Springer Medizin Verlag GmbH, ein Teil von Springer Nature 2021

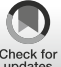

\section{Update Thoraxpathologie 2021 - Bericht der Arbeitsgemeinschaft}

\author{
Sabina Berezowska ${ }^{1}$ Peter Boor ${ }^{2}$. Danny Jonigk ${ }^{3}$. Verena Tischler ${ }^{4}$ \\ ${ }^{1}$ Institut universitaire de pathologie, Centre hospitalier universitaire vaudois (CHUV) et Université de \\ Lausanne, Lausanne, Schweiz \\ ${ }^{2}$ Institut für Pathologie, Universitätsklinikum Aachen, Rheinisch-Westfälische Technische Hochschule \\ Aachen, Aachen, Deutschland \\ ${ }^{3}$ Institut für Pathologie, Medizinische Hochschule Hannover, Hannover, Deutschland \\ ${ }^{4}$ Institut für Pathologie, Universitätsklinikum Bonn, Bonn, Deutschland
}

Dieses Jahr war ein besonderes Jahr. Doch trotz der Reisebeschränkungen und der Herausforderungen in der Organisation von Kongressen konnte die Arbeitsgemeinschaft Thoraxpathologie wie gewohnt zweimal tagen - wenn auch nur in virtueller Form. Obwohl die sozialen Interaktionen und Diskussionen dadurch naturgegeben eingeschränkt waren, war der wissenschaftliche Austausch sehr wichtig und von hochkarätigen Vorträgen dominiert.

Die weiterhin andauernde COVID-19Pandemie war sowohl während der Frühjahrstagung ein dominantes Thema und wurde auch während der virtuellen Pathologietage neben der regulären Sitzung der AG als eigenständige Session im Hauptprogramm am Freitag behandelt.

\section{Virtuelles Frühjahrstreffen}

Das Frühjahrstreffen fand am 5. Februar von 15 bis 19 Uhr in virtueller Form statt und wurde von Sabina Berezowska (Lausanne, Schweiz) und Verena Tischler (Bonn) organisiert. Die Veranstaltung war auch für Nicht-DGP-Mitglieder offen und sehr gut besucht, mit insgesamt 96 angemeldeten Teilnehmern aus Deutschland, Österreich, der Türkei, der Schweiz und den USA.

Die erste Session beschäftigte sich mit einem Update zu COVID-19. Zunächst berichtete Alexandar Tzankov (Basel, Schweiz) darüber, was In-situ-Untersuchungen am Autopsiegewebe zur Untersuchung des Pathomechanismus von letaler COVID-19 beitragen können und bereits bisher beigetragen haben [3]. Im Anschluss berichtete Christopher
Werlein (AG Lungenforschung, Institut für Pathologie $\mathrm{MHH}$ ) ebenfalls zu COVID-19 aus der Perspektive eines Pathologen und fasste die die hochkarätigen Publikationen und aktuelle Untersuchungen aus Hannover zusammen [1]. Abschließend gab Viktor Kölzer (Zürich, Schweiz) eine Zusammenfassung der Studie, in der das Team zwischen Liestal (Kirsten Mertz), Zürich (Viktor Koelzer) und Trento (Francesca Demichelis) zwei distinkte immunpathologische Profile in Autopsielungen von COVID-19-Verstorbenen identifiziert haben [7]. Durch die Sitzung führte Sabina Berezowska. Wie zu erwarten gab es viel Diskussionsbedarf, der die anschließende Pause verkürzte.

Die zweite Session zum Thema „Update Molekularpathologie und translationale Lungenpathologie" wurde durch Verena Tischler geleitet. Sabine Merkelbach-Bruse (Köln) berichtete über die Fortschritte in der molekularen Diagnostik des Verbundprojekts Nationales Netzwerk genomische Medizin (nNGM) und hier insbesondere über innovative genomische Panels, welche insbesondere den Patient:innen neben zielgerichteten Standardtherapien früh und strukturiert Zugang zu klinischen Studien ermöglichen. Außerdem legte Sabine Merkelbach-Bruse die Harmonisierung der molekularen Diagnostik an den nNGM-Netzwerkzentren und die besonderen qualitätssichernden Maßnahmen anschaulich dar [4]. Weiter ging es mit Sonja Loges (Hamburg und Mannheim), die die Studie ihrer Arbeitsgruppe zu den typischen und atypischen EGFR-Mutationen vorstellte. In dieser Studie hat Sonja Loges mit ihrem Team eine beeindrucken- 
de präklinische Pipeline zur funktionellen Charakterisierung von klinisch unklaren EGFR-Varianten der innerhalb des Netzwerks Genomische Medizin und des nNGMs aufgefunden Mutationsspektren zur Therapieentscheidung dargelegt. Im Anschluss sprach Jürgen Wolf (Köln) über die klinische Seite der MET-Inhibitoren beim nichtkleinzelligen Lungenkarzinom (NSCLC) und ging ausführlich auf die molekularen MET-Alterationen und die Bedeutung ihrer präzisen molekularpathologischen Bestimmung für die klinische Therapie ein. Martin Sebastian (Frankfurt) sprach über die bahnbrechenden Therapieerfolge bei NSCLC-Patient:innen mit KRAS-Mutationen und betonte die Wichtigkeit der präzisen Variantenbestimmung und des exakten Reportings im molekularpathologischen Befund für die beste Therapiewahl.

Zum Abschluss des wissenschaftlichen Programms gab Alexander Marx (Mannheim) ein Update zu Mediastinaltumoren bereits als Vorgriff auf die später im Jahr erschienene WHO-Klassifikation und unter Einbeziehung ganz aktueller, noch nicht publizierter Daten. Sabina Berezowska schloss das Programm ab mit einem 10minütigen Update zur vom August 2020 verschobenen und dann letztendlich eine Woche vor dem Frühjahrstreffen nachgeholten „World Conference on Lung Cancer 2020" der International Association for the Study of Lung Cancer (IASLC), die, anstatt in Singapur, ebenfalls virtuell durchgeführt hat werden müssen.

Die Mitgliederversammlung wurde aufgrund des virtuellen Formates stark verkürzt. In der angesetzten Viertelstunde wurde Verena Tischler zur stellvertretenden Sprecherin der AG gewählt. Zudem wurde die Anzahl der Beiratsmitglieder diskutiert und eine aktuelle Anzahl von 9 Mitgliedern inklusive eines kooptierten Mitglieds als konform mit den aktuellen Statuten der AG verifiziert. Der Vorschlag, dass zukünftig ein Ergebnisprotokoll der Mitgliedsversammlungen durch Sprecher:in oder Stellvertreter:in geführt wird, wurde von den Mitgliedern angenommen. Den Abschluss der Mitgliederversammlung bildete eine Diskussion zu den Vorschlägen der eingeladenen Referenten für die "Virtuellen Pathologietage 2021" und eine Diskussion zum Thema nNGM.
Virtuelle Pathologietage 2021

\section{AG-Sitzung}

Im Pandemiejahr 2021 wurde die reguläre Jahrestagung der DGP ausgesetzt und durch die „Virtuellen Pathologietage 2021" ersetzt.

Die Sitzung der AG Thoraxpathologie fand am ersten Kongresstag, dem 8. Juni, von 16 bis 18 Uhr als Livesitzung statt. Die Resonanz war hervorragend, mit einem Maximum von zeitweise bis zu 99 eingeloggten Teilnehmern. Während der Onlinepräsentationen konnten über die Chatfunktion fortlaufend Fragen gestellt werden, die während der anschließenden Diskussion mündlich oder aus Zeitgründen anschließend daran ebenfalls im Chat beantwortet wurden. Dies ermöglichte trotz des virtuellen Formates eine angeregte Diskussion.

Die Sitzung wurde durch die beiden hochkarätigen Gastvorträge eröffnet. Zunächst berichtet Katrien Grunberg aus Nijmegen (Niederlande) zur Gefäßpathologie der Lunge, die aktuell vor allem durch die Corona-Pandemie ins Scheinwerferlicht gerückt ist. Ihr Vortrag „A practical approach to vasculopathy in pulmonary hypertension" schloss auch Kommentare zur vaskulären Pathologie bei COVID19 mit ein. Anschließend referierte Philipp Ströbel aus Göttingen die aktuellen Erkenntnisse zu neuroendokrinen Neoplasien des Thymus und der Lunge mit der spezifischen Frage, ob es sich um den gleichen Tumor in 2 Organen handelt [2]. Die Unterschiede der Neoplasien abhängig von der Lokalisation waren frappierend.

Der zweite Teil der Sitzung war für eingereichte Beiträge reserviert. Florian Länger (Hannover) gab einen sehr umfassenden Überblick zu interstitiellen Lungenerkrankungen im Säuglings- und Kindesalter, der sicher einen längeren Slot verdient hätte [5]. Ralf Marienfeld (UIm) trug die Ergebnisse zur räumlichen Verteilung der Immuncheckpoint-Proteine in unterschiedlichen morphologischen Subtypen von Adenokarzinomen der Lunge vor [6]. Philip Bischoff (Berlin) berichtete zur Charakterisierung des Tumormikromilieus in Adenokarzinomen der Lunge mittels Einzelzell-RNA-Sequenzierung. Sylvia Lohfink-Schumm (Bonn) sprach zur Mutati- onslandschaft von nichtkleinzelligen Lungenkarzinomen mit MET-Genalterationen. Zuletzt fasste Sabina Berezowska in einem 5-minütigen Vortrag die als Poster präsentierten Einreichungen aus der AG Thoraxpathologie zusammen. Die Posterpräsentationen waren als „besprochene Powerpoint-Datei" abrufbar und technisch und inhaltlich von hervorragender Qualität.

Aufgrund der fortgeschrittenen Zeit und technischer Schwierigkeiten mit einem zwar pünktlichen, aber unangenehm abrupten Abschluss der Sitzung für die zugeschalteten Kongressteilnehmer konnte die Mitgliederversammlung nicht adäquat durchgeführt werden. Wir freuen uns umso mehr auf das nächste Treffen im Rahmen der Frühjahrstagung 2020.

\section{COVID-19-Sitzung}

Bereits bei der Organisation der Jahrestagung 2020 kam aus der AG Thoraxpathologie der anschließend umgesetzte Vorschlag, eine zusätzliche Sitzung zu dem Thema SARS-CoV-2 mit dem Schwerpunkt Diagnostik und histopathologische Veränderungen zu organisieren. Dies wurde auch dieses Jahr beibehalten. Das Hauptprogramm der "Virtuellen Pathologietage 2021" am Freitag den 11. Juni 2021 wurde somit mit dem Thema COVID-19 eröffnet. Nach der Keynote Lecture "Genomic Epidemiology of SARS-CoV-2: From Variants of Concern to Understanding Population Transmission Chains" durch Alexander Dilthey (Düsseldorf) folgte ein Update zu COVID-19 vonseiten der Pathologie. Durch die Sitzung führten Danny Jonigk (Hannover) und Peter Boor (Aachen).

Eröffnet wurde die Sitzung von Peter Boor, der das - immer noch international einzigartige - deutsche Register für COVID19-Obduktionen und das deutsche Netzwerk für Autopsien bei Pandemien (Defeat Pandemics) vorstellte, erste Ergebnisse der Datenauswertung präsentierte und wiederum für das wichtige kooperative Miteinander aller Beteiligten warb. Darauf folgte Tobias Welte (Hannover), der eine beeindruckende Synopse der Entwicklung der COVID-19-Pandemie aus epidemiologischer, virologischer und klinischer Sicht und - immer gestützt auf aktuelle Studien - einen Ausblick für die wahrscheinliche weitere Entwicklung von COVID-19 in 
Deutschland und der Welt gab. Danach führte Leif Erik Sander (Berlin) äußerst kompetent und umfassend durch die Aufschlüsselungen der beeinträchtigten Immunantwort bei schweren (systemischen) COVID-19-Verläufen.

In der Folge stellte Danny Jonigk erste umfassende Daten zu kompartimentenspezifischen Alterationen bei COVID-19Beteiligungen von Herz und Lunge vor und arbeitete insbesondere die angiozentrische Pathogenese der Erkrankung heraus. Im Anschluss referierte Michael H. Roehrl (New York, USA), wie eine SARS-CoV2-Infektion die Abwehrreaktion des Wirtes signifikant verändern und autoimmunologische Mechanismen aktivieren kann. Darüber hinaus stellte er erste Verbindungen der vorbeschriebenen Mechanismen zum "Long-COVID-Syndrom" her. Abgerundet wurde die Sitzung von Konrad Steinestel (Bundeswehrkrankenhaus Ulm), der sich umfassend mit den klinischen, bildgebenden, serologischen, histopathologischen Veränderungen des (pulmonalen) "LongCOVID-Syndroms" befasste und wie auch sein Vorredner die wahrscheinliche Rolle fehlaktivierter (auto-)immunologischer Mechanismen kompetent herausarbeitete.

Während der „Virtuellen Pathologietage" wurde zum Thema Thoraxpathologie auch in anderen Sitzungen referiert. Die Beiträge werden hier gelistet.

Vortrag im Rahmen der Sitzung der AG Kopf-Hals-Pathologie

- High-resolution mass spectrometry identifies new immunohistochemical markers to differentiate primary squamous cell carcinomas of the lung and metastasis of squamous cell carcinomas of the head and neck (AG05.02, A. Richter et al.)

Vortrag im Rahmen der Sitzung der AG Molekularpathologie

- Clonal hematopoiesis in lung cancer patients-detection of false-positive findings in liquid biopsies by parallel sequencing. (AG14.01, J. Fassunke et al.)
Vortrag im Rahmen der Bewerbung um den DGP-Promotionspreis

- Solution to the challenges of automated immune cell detection in the lung_anthracosis (DGP01.10, P. Zens)

Vorträge im Rahmen der Bewerbung um den DGP-Forschungspreis

- SARS-CoV-2 infects and replicates in cells of the human endocrine and exocrine pancreas (DGP07.01, T. FE Barth)

- Deciphering the proteome of COVID19-a multi-organ proteomic profiling of COVID-19 autopsies (DGP07.02, L. Schweizer)

Um auch alle als Poster präsentierten Beiträge zum Thema Thoraxpathologie und COVID-19 zu würdigen, sind sie im Nachfolgenden aufgeführt. Nähere Informationen können dem Abstractband entnommen oder direkt von den Autoren angefordert werden.

- Potential role of Tenascin C (TNC) in human non-small cell lung cancer progression (P02.01, M. Schlensog et al.)

- Cancer-associated fibroblasts regulate kinase activity in MPM cell lines and thereby dictate cell fate and behavior (P02.02, A. Mathilakathu et al.)

- Influence of DNA Damage Repair, TP53-Network and Metallothionein on the Susceptibility to Cisplatin in Malignant Pleural Mesothelioma (P02.03, S. Borchert et al.)

- Comprehensive molecular profiling of malignant pleural mesothelioma: a proof-of-concept study (P02.04, H. Goldschmid et al.)

- Cancer associated fibroblasts and malignant pleural mesothelioma: revealing the role of the tumor microenvironment on tumor progression (P02.05, A. Nath et al.)

- Digital gene expression analysis reveals differences in immunogenicity based on subtypes of malignant pleural mesothelioma (P02.06, M. Wessolly et al.)

- Mitogen signal associated pathways energy metabolism regulation as well as mediation of tumor immunogenicity play important roles in cellular response of pleural mesotheliomas to cisplatin-based treatment (P02.07, A. Mathilakathu et al.)

- Cancer-Associated Fibroblasts and their Influence on Cancer Progression and Motilityin Malignant Pleural Mesothelioma (P02.08, A. Mathilakathu et al.)

- Autopsy findings after long-term treatment of COVID-19 patients with microbiological correlation (PCov.01, K. Evert et al.)

- All-Body-Cavity (ABC)-Scopy as a New Approach for Minimal Invasive Post Mortem Examination (PCov.02, L. Rentschler et al.)

- COVID-19 in a patient with active miliary tuberculosis: clinical outcome and histopathology of lung parenchyma (PCov.03, S. Opitz et al.)

- How COVID-19 pathology data contribute to research and patient treatment (PCov.04, L. Domke et al.)

- A sensitive protocol for SARS-CoV-2 RNA screening in diagnostic archives (PCov.05, S. Villwock et al.)

- COVID-19: Preliminary results from a Swab-Test-Study to detect SARS-CoV2 on personal protective equipment of autopsy staff at four German centers (PCov.06, S. Dintner et al.)

- Results from the Augsburg-COVID-19autopsy series (PCov.07, T. Schaller et al.)

Präsentiert im Rahmen der Poster der Kinder- und Fetalpathologie:

- ABCA3 deficiency - Aggravating factor for neonatal respiratory distress syndrome (RDS) in sepsis? (P03.01, C. Jayasinghe et al.)

Präsentiert im Rahmen der Poster der Uropathologie:

- Gene expression analysis of prostate cancer lung metastases (P11.10, K. Hempel et al.)

Präsentiert im Rahmen der Poster der Molekularpathologie:

- Immune cell biomarkers in lung adenocarcinoma: inter- and intra-tumor heterogeneity (P14.02, J. Budczies et al.)

- Detecting EGFR T790M resistance mutation in plasma at progression on 


\section{Berichte der Arbeitsgemeinschaften}

TKI therapy using ultrasensitive digital PCR (P14.15, S. Isaksson et al.)

- Comparison of two different targeted NGS panels for molecular diagnosis of non-small cell lung cancer: ArcherDx versus ThermoFisher Scientific. (P14.16, M. Wetz et al.)

Somit kann die AG Thoraxpathologie auch im zweiten Corona-Jahr trotz aller Einschränkungen auf ein ereignisreiches und erfolgreiches Jahr zurückblicken. Die nächste Sitzung der AG soll während des Frühjahrstreffens 2022 in Bonn als Hybridveranstaltung stattfinden und steht unter der Leitung von PD Dr. Verena Tischler. Wir freuen uns schon sehr darauf!

\section{Korrespondenzadresse}

\section{Sabina Berezowska}

Institut universitaire de pathologie, Centre hospitalier universitaire vaudois (CHUV) et Université de Lausanne Rue du Bugnon 25, 1011 Lausanne, Schweiz Sabina.Berezowska@chuv.ch

\section{Einhaltung ethischer Richtlinien}

Interessenkonflikt. S. Berezowska, P. Boor, D. Jonigk und V. Tischler geben an, dass kein Interessenkonflikt besteht.

Für diesen Beitrag wurden von den Autoren keine Studien an Menschen oder Tieren durchgeführt. Für die aufgeführten Studien gelten die jeweils dort angegebenen ethischen Richtlinien.

The supplement containing this article is not sponsored by industry.

\section{Literatur}

1. Ackermann $M$, Werlein $C$, Langer $F$ et al (2021) COVID-19: effects on the lungs and heart. Pathologe 42:164-171

2. Bohnenberger $H$, Ströbel P(2021) Recent advances and conceptual changes in the classification of neuroendocrine tumors of the thymus. Virchows Arch 478:129-135

3. Bösmüller $H$, Matter $M$, Fend $F$ et al (2021) The pulmonary pathology of COVID-19. Virchows Arch 478:137-150

4. Heydt $C$, Wölwer CB, Velazquez Camacho 0 et al (2021) Detection of gene fusions using targeted next-generation sequencing: a comparative evaluation. BMCMed Genomics 14:62

5. Langer F, Werlein C, Soudah B et al (2021) Interstitial lung disease in infancy and early childhood. Pathologe 42:25-34
6. Müller S, Mayer S, Möller P et al (2021) Spatial distribution of immune checkpoint proteins in histological subtypes of lung adenocarcinoma. Neoplasia 23:584-593

7. Nienhold R, Ciani Y, Koelzer VH et al (2020) Two distinct immunopathological profiles in autopsy lungs of COVID-19. Nat Commun 11:5086 